\title{
COVID-19 and the kidney; mechanisms of tubular injury by SARS-CoV-2
}

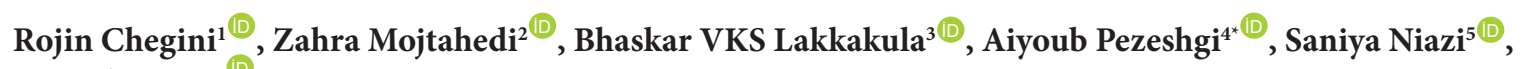 \\ Hamid Nasri ${ }^{6,7}$ \\ ${ }^{1}$ Isfahan University of Medical Sciences, Isfahan, Iran \\ ${ }^{2}$ Department of Health Care Administration and Policy, School of Public Health, University of Nevada, Las Vegas, Las Vegas, Nevada, USA \\ ${ }^{3}$ Department of Zoology, Guru Ghasidas Vishwavidyalaya, Bilaspur, India \\ ${ }^{4}$ Department of Internal Medicine and Zanjan Metabolic Disease Research Center, School of Medicine, Zanjan University of Medical Sciences, Zanjan, \\ Iran \\ ${ }^{5}$ Comprehensive Hematology Oncology LLC. 5000 Park St N \# 1017, St. Petersburg, Fl, USA \\ ${ }^{6}$ Infectious Diseases and Tropical Medicine Research Center, Isfahan University of Medical Sciences, Isfahan, Iran \\ ${ }^{7}$ Nickan Research Institute, Isfahan, Iran
}

\section{A R T I C L E I N F O}

Article Type:

Review

\section{Article History:}

Received: 7 September 2020

Accepted: 25 October 2020

ePublished: 26 November 2020

Keywords:

Chronic kidney disease,

COVID-19, Acute respiratory

distress syndrome, SARS-

CoV-2, Angiotensin-converting

enzyme II, Hemodialysis, Acute

renal failure, End-stage renal

disease, Acute kidney injury,

\begin{abstract}
A B S T R A C T
Coronavirus disease 2019 (COVID-19) is an ongoing pandemic, reported to cause asymptomatic to severe disease and eventually death. Multi-organ failure and death in patients with severe COVID-19 is associated with increased release of pro-inflammatory cytokines into the blood stream. Renal impairment is reported in a significant proportion of COVID-19 patients and is associated with high mortality. Acute kidney injury (AKI) is multifactorial and involving overlapping pathogenic mechanisms. This review updates the reader of recent publications dealing with the mechanisms underlying AKI in patients with COVID-19. A full understanding of all the possible ways in which the system plays its role in AKI is still a matter of research. Further studies are warranted to better understand the causes of AKI in COVID-19 patients.
\end{abstract}

Implication for health policy/practice/research/medical education:

Acute kidney injury (AKI) is more common in severely ill patients with COVID-19. AKI is strongly correlated with the occurrence of respiratory failure disease severity. Acute kidney injury in COVID-19 patients conferred a poor prognosis and outcomes.

Please cite this paper as: Chegini R, Mojtahedi Z, Lakkakula BVKS, Pezeshgi A, Niazi S, Nasri H. COVID-19 and the kidney; mechanisms of tubular injury by SARS-CoV-2. J Renal Inj Prev. 2021; 10(1): e08. doi: 10.34172/jrip.2021.08.

\section{Introduction}

The disease caused by a SARS-CoV-2 infection, which is called coronavirus disease 2019 (COVID-19), is an ongoing pandemic. Although the catastrophic pulmonary effects of COVID-19 have been well documented, damage to other organ systems, such as the heart, kidneys, and liver, has also been reported (1). The predominant clinical symptoms of COVID-19 are similar to those of common cold and flu, including dry cough, dyspnea, myalgia pneumonia, fatigue and fever (2). Clinical manifestation of COVID-19 has been reported to range from asymptomatic to severe (hyperinflammatory shock) and eventually death (3). Numerous studies have shown that increased pro-inflammatory cytokines could release into the bloodstream and cause a "cytokine storm" leading to multi-organ failure and death in patients with severe COVID-19. Further, acute respiratory distress syndrome (ARDS) is the most significant pulmonary complication severely infected patients (1-3).

Renal impairment is also reported in a significant 
proportion of COVID-19 patients and is associated with high mortality (2). Although the occurrence of acute kidney injury (AKI) and its effect on COVID-19 are not fully understood, the assessment of renal function during SARS-CoV-2 infection is of particular interest (4). AKI is more common in people with more severe disease, principally critically ill patients in intensive care units. Hence, AKI is considered as a marker of disease severity and a negative prognostic factor for survival (5). A recent study from the United States, which included the majority of African American patients, reported an overall frequency of AKI of $28 \%$, of which more than $50 \%$ of the cases required emergency dialysis (6). Another study from the USA found that the overall AKI frequency in hospitalized patients ranged from $28 \%$ to $46 \%$, while initial reports from China showed that the overall AKI frequency increased from $0.5 \%$ to $29 \%$ (7). It is generally accepted that the mortality rate of SARS-CoV-2 cases with $\mathrm{AKI}$ is thought to be significant, ranging from $8 \%$ to $23 \%$. As development of AKI in SARS-CoV-2 individuals is a major predictive factor for poor survival (8), the physicians should pay specific attention to SARS-CoV-2 infected patients manifesting AKI. As AKI is multifactorial involving with overlapping pathogenic mechanisms, this review updates the reader of recent publications dealing with the mechanisms underlying AKI in patients with COVID-19 (8).

\section{Methods}

Data were collected in Scopus, EBSCO, Google Scholar, Web of Science and PubMed databases. The keywords used include SARS-CoV-2, COVID-19, chronic kidney disease, acute respiratory distress syndrome, angiotensinconverting enzyme II, hemodialysis, acute renal failure, remdesivir, end-stage renal disease, acute kidney injury, cellular transmembrane serine protease 2 and renal injury. In addition to search terms, we limit data collection to research and review articles written in English only.

\section{Mechanisms of acute renal injury in SARS-CoV-2}

Since AKI is associated with ARDS during SARSCoV-2 infection, AKI can be attributed to a variety of factors, including hemodynamic changes, such as fluid overload and systemic congestion, harmful mechanical ventilation strategies and the development of sepsis. Further, several studies have highlighted the importance of an inflammatory/immune-mediated response with the release of high levels of harmful circulating mediators that can interact with kidney-inhabiting cells, leading to endothelial dysfunction and tubular injury. Given the frequency of their occurrence and the fact that these etiologies associated with acute kidney injury will be discussed briefly (2-8).

Kidney is a specific target for SARS-CoV-2 infection Since SARS-CoV-2 RNA was detected in the blood and urine samples of patients with confirmed SARS-CoV-2 infections, there is a possibility of direct invasive effect on the glomerular, tubular and vascular renal cells by this virus. SARS-CoV-2 entry into cells is mediated by its surface spike protein, which binds to its receptor human ACE2 and cellular transmembrane serine protease 2 (TMPRSS2) receptors (9). Several lines of evidence demonstrated that the ACE2 and TMPRSS2 are significantly expressed in the proximal tubule of nephron (10). A measurable level of TMPRSS2 was identified in the S3 segment of the proximal tubule (11). ACE2 is abundantly present in the renal cells, such as parietal epithelium of Bowman's capsule, collecting ducts, thick ascending limb of Helen, podocytes, proximal cell brush border and mesangial cells (9). Subsequent to the binding of coronavirus to the receptor of ACE2 or co-receptor of TMPRSS2, the virus uses the cellular body endocytosis system to join the membrane and then enter the cell. These findings indicate that the kidneys are one of the targets for COVID-19 (12). Additional studies also showed that the virus is capable of inducing acute tubular injury following proximal tubular invasion. A more recent investigation showed that the NP (nucleocapsid) antigen of coronavirus was deposited in the cytoplasm of renal tubular cells in patients who died from COVID-19 (13). Another possible mechanism of renal involvement is the deposition of antibodies or immune complexes caused by the infection (14). In addition, activating the immune system after being infected with the virus cannot eradicate the virus, but it can damage or alter normal body cells (15).

Cytokine storm syndrome secondary to SARS-CoV-2 infection

Elevated blood concentration of cytokines and proinflammatory mediators like IL-6, IL-7, IL10, IL2, TNFa, MCP1 and GSCF induce the cytokine storm which lead to renal, heart and lung damage in severely infected SARS-CoV-2 patients (16). Inflammation associated with a cytokine storm begins locally and spreads to the entire body through systemic circulation (17). Cytokine storm syndrome triggers systemic endothelial injury, which results in acute renal damage following raised vascular leaking, intra-renal inflammation, and volume depletion. In addition, decreased renal perfusion eventually leading to cardiorenal syndrome involving intra-abdominal hypertension, pleural effusions, volume depletion due to loss to fluid from the third space, and edema. Among interleukins (ILs), IL-6 plays a main role in cytokine storm syndrome. Recent studies have detected that patients with ARDS had the high blood concentration of interleukin 6. Accordingly, ARDS may stimulate additional injury to renal tubular cells in renal medulla by hypoxic damage (18). It is important to note that the baseline renal function is a determinant factor to develop acute renal damage. Patients with elevated serum creatinine are more likely to worsen pre-existing renal failure than patients with 
normal kidney function. After the onset of cytokine storm syndrome and/or inflammation, compensatory repair processes occur to restore the affected tissues and organ function. Severe inflammation and injury are followed by healing with fibrosis and tissue destruction and finally by permanent organ dysfunction (17). In this regard, it was initially believed that patients with chronic renal failure could be protected from cytokine storm syndrome, but it has recently been found that the SARS-CoV-2 infected patients, and in particular patients with end-stage renal disease, have higher morbidity and mortality (19).

Hypoxia causes kidney malfunction during SARSCoV-2 infection

Tissue hypoxia has been proposed as an important factor in the pathophysiology of AKI. Limited the oxygen supply to the kidney tissue makes the kidney susceptible to hypoxia and accelerates the deterioration of renal function. The coexistence of hypoxia and inflammation may further direct the inflammatory response toward worse outcome in infection rather than tissue recovery (20). Considering that severe hypoxia is a classic feature of severe SARS-CoV-2 infection, it is very likely that a system that induces hypoxia (HIF) will be involved, which may affect the inflammatory response and the outcome of the kidneys (21). In support of this, involvement of the HIF pathway during AKI has been shown in various kidney disease models (22).

\section{Hypercoagulability and thrombosis in COVID-19}

Patients with COVID-19 ARDS exhibited an abnormal coagulation parameter and poor prognosis (23). Laboratory results show that the elevated $\mathrm{D}$-dimer on admission or marked increase in D-dimer levels during the illness is associated with high mortality (24). In addition, antiphospholipid antibodies and infarcts in vascular regions have been reported in patients with COVID-19 (25). Retention of metabolic toxins due to renal dysfunction leads to a state of hypercoagulation during infections. However, whether AKI affects hypercoagulability and thromboembolism in COVID19 patients is unclear (23-25).

\section{Aggravating factors of acute kidney injury}

Acute renal injury is more common in severely ill patients with COVID-19 (9). Various factors such as nephrotoxic agents, hypotension, sepsis, dehydration and hypoxemia play a role in the development of acute kidney injury (13). In addition, medications, including antiviral substances, antibiotics and NSAIDs are contributing factors for worsening of acute renal injury (13). Patients with severe COVID-19 often exhibit electrolyte and acidbase imbalance, diarrhea, disseminated intravascular coagulation, and heart failure. Acute kidney injury and concomitant endothelial damage, glomerular hypertension, interstitial infiltration, and fibrosis are major factors leading to chronic renal failure after an episode of acute kidney injury (26). The chronic conditions that affect the immune system include heart disease; asthma, lupus, and diabetes increase the burden of morbidity and mortality associated with COVID-19 (27). Further, acute kidney injury (AKI) in COVID-19 patients is associated with elevated creatine phosphokinase (CPK) and rhabdomyolysis (28). In the process of rhabdomyolysis, muscle damage causes a massive release of myoglobin in the kidneys, a direct toxic substance for renal tubular cells. Consequently, tubular obstruction and vasoconstriction increase renal impairment by myoglobin. In addition, myoglobin stimulates hyperactivation of the reninangiotensin-aldosterone system and reduces nitric oxide (NO) levels, causing renal hypoperfusion and acute tubular injury (29).

Kidney damage from SARS-CoV-2 infection is not only a result of the direct effects of the virus, but also of the treatment modalities. Binois et al, showed that the patients treated with lopinavir/ritonavir developed acute renal failure (30). Lopinavir/ritonavir combination use has been associated with acute interstitial nephritis symptoms in COVID 19 patients. However continuing this drug combination is also associated with renal proximal tubular damage (30). Remdesivir is a broad-spectrum anti-viral agent that is administered using a vehicle called cyclodextrin. As cyclodextrin causes nephrotoxicity and impairment of renal function, there are concerns regarding the use of the drug in patients with pre-existing kidney disease (31). Severe Covid-19 patients treated with compassionate-use remdesivir showed acute renal failure only in patients requiring mechanical ventilation (32). Hence, monitoring of renal function should be considered during remdesivir therapy.

\section{SARS-CoV-2-associated renal tubulopathy}

The presence of hematuria and albuminuria in cases of SARS-CoV-2, together with viral RNA in urine samples, also shows the intense tropism of the SARS-CoV-2 for renal tissues (33). Histopathological studies in COVID-19 patients with renal dysfunction, showed proximal acute tubule injury (ATI), the occlusion of microvascular lumens, endothelial damage, as well as glomerular and vascular changes (34). In addition, intermittent hemosiderin granules and pigmented casts were identified (34). Further, co-occurrence of lymphocytes infiltration with acute renal tubular necrosis in the interstitial area was noted (35). In some cases, renal proximal tubule dysfunctions with proteinuria and aminoaciduria have been found (36). In this study, renal proximal tubule dysfunctions were found to be independent of treatmental modality and glomerular proteinuria. Laboratory findings of SARS-CoV-2 individuals without history of renal disease, showed a condition equaling to Fanconi syndrome before presenting with acute renal failure (37). 
Impact of acute kidney injury on survival of COVID-19 Initial studies from China reported a raised occurrence of AKI in below $7 \%$ of inpatient admissions and around $23 \%$ in adult cases who admitted to ICUs (38). The subsequent studies revealed that AKI increases the mortality of adult patients with SARS-CoV-2 $(39,40)$. Higher incidence of AKI was documented in severely ill COVID-19 patients (41). A recent study from Pakistan indicated that the renal function impairment at all levels of disease severity which well correlated with plasma creatinine, urea and potassium (42). Analysis of data from the international HOPE-Registry, indicated that the kidney insufficiency at the time of admission is related to a larger amount of morbidity and mortality (19). A systematic review and meta-analysis suggested that the kidney dysfunction is common among patients with COVID-19, and patients who develop AKI have severe disease and inferior outcomes and increased mortality (4). Further, it is also reported that the AKI occurs frequently in Covid-19 patients and $\mathrm{AKI}$ in temporal association with respiratory failure is associated with a poor prognosis (43).

\section{Conclusion}

In conclusion, AKI is more common in severely ill patients with COVID-19. AKI is strongly correlated with the occurrence of respiratory failure disease severity. AKI in COVID-19 patients conferred a poor prognosis and outcomes. A full understanding of all the possible ways in which the system plays its role in AKI is still a matter of research. Further studies are warranted to better understand the causes of AKI in COVID-19 patients.

\section{Authors' contribution}

Primary draft by RG, HN and LBVKS, ZM, AP and SN edited the paper. RG, HN and AP finalized the manuscript. All authors read and signed the final paper.

\section{Conflicts of interest}

The authors declared no potential conflicts of interest with respect to the research, authorship, and/or publication of this article.

\section{Ethical considerations}

Ethical issues (including plagiarism, data fabrication, double publication) have been completely observed by the authors.

\section{Funding/Support}

None.

\section{References}

1. Zaim S, Chong JH, Sankaranarayanan V, Harky A. COVID-19 and multiorgan response. Curr Probl Cardiol. 2020;45(8):100618. doi:10.1016/j.cpcardiol.2020.100618

2. Ren X, Wei X, Li G, Ren S, Chen X, Zhang T, et al. Multiple expression assessments of ACE2 and TMPRSS2 SARS-CoV-2 entry molecules in the urinary tract and their associations with clinical manifestations of COVID-19. bioRxiv. 2020:2020.05.08.083618. doi:10.1101/2020.05.08.083618

3. Bajwa H, Riaz Y, Ammar M, Farooq S, Yousaf A. The Dilemma of Renal Involvement in COVID-19: A Systematic Review. Cureus. 2020;12:e8632. doi:10.7759/cureus.8632

4. Robbins-Juarez SY, Qian L, King KL, Stevens JS, Husain SA, Radhakrishnan J, et al. Outcomes for patients with COVID-19 and acute kidney injury: a systematic review and meta-analysis. Kidney Int Rep. 2020;5:1149-60. doi:10.1016/j.ekir.2020.06.013

5. Durvasula R, Wellington T, McNamara E, Watnick S. COVID-19 and kidney failure in the acute care setting: our experience from seattle. Am J Kidney Dis. 2020;76:4-6. doi:10.1053/j.ajkd.2020.04.001

6. Chan L, Coca SG. Acute kidney injury in the time of COVID-19. Kidney360. 2020;1:588-90. doi:10.34067/ kid.0003722020

7. Sharma P, Uppal NN, Wanchoo R, Shah HH, Yang Y, Parikh $\mathrm{R}$, et al. COVID-19-associated kidney injury: a case series of kidney biopsy findings. J Am Soc Nephrol. 2020;31(9):194858. doi:10.1681/asn.2020050699

8. Shao M, Li X, Liu F, Tian T, Luo J, Yang Y. Acute kidney injury is associated with severe infection and fatality in patients with COVID-19: a systematic review and metaanalysis of 40 studies and 24,527 patients. Pharmacol Res. 2020;161:105107. doi:10.1016/j.phrs.2020.105107

9. Martinez-Rojas MA, Vega-Vega O, Bobadilla NA. Is the kidney a target of SARS-CoV-2? Am J Physiol Renal Physiol. 2020;318:F1454-f62. doi:10.1152/ajprenal.00160.2020

10. Chen QL, Li JQ, Xiang ZD, Lang Y, Guo GJ, Liu ZH. Localization of cell receptor-related genes of SARS-CoV-2 in the kidney through single-cell transcriptome analysis. Kidney Dis (Basel). 2020;6:258-70. doi:10.1159/000508162

11. Ransick A, Lindström NO, Liu J, Zhu Q, Guo JJ, Alvarado GF, et al. Single-cell profiling reveals sex, lineage, and regional diversity in the mouse kidney. Dev Cell. 2019;51:399-413.e7. doi:10.1016/j.devcel.2019.10.005

12. Asgharpour M, Zare E, Mubarak M, Alirezaei A. COVID-19 and kidney disease: update on epidemiology, clinical manifestations, pathophysiology and management. J Coll Physicians Surg Pak. 2020;30:19-25. doi: 10.29271/ jcpsp.2020.Supp1.S19

13. Qian JY, Wang B, Liu BC. Acute kidney injury in the 2019 novel coronavirus disease. Kidney Dis. 2020;6:318-23. doi:10.1159/000509086

14. Meena P, Bhargava V, Rana DS, Bhalla AK, Gupta A. COVID-19 and the kidney: a matter of concern. Curr Med Res Pract. 2020;10:165-8. doi:10.1016/j.cmrp.2020.07.003

15. Batlle D, Soler MJ, Sparks MA, Hiremath S, South AM, Welling PA, et al. Acute kidney injury in COVID-19: emerging evidence of a distinct pathophysiology. J Am Soc Nephrol. 2020;31:1380-3. doi:10.1681/asn.2020040419

16. Costela-Ruiz VJ, Illescas-Montes R, Puerta-Puerta JM, Ruiz C, Melguizo-Rodríguez L. SARS-CoV-2 infection: The role of cytokines in COVID-19 disease. Cytokine Growth Factor Rev. 2020;54:62-75. doi: 10.1016/j.cytogfr.2020.06.001

17. Gagliardi I, Patella G, Michael A, Serra R, Provenzano M, Andreucci M. COVID-19 and the kidney: from epidemiology to clinical practice. J Clin Med. 2020;9:2506. 
doi: $10.3390 / \mathrm{jcm} 9082506$

18. Bajwa H, Riaz Y, Ammar M, Farooq S, Yousaf A. The dilemma of renal involvement in COVID-19: a systematic review. Cureus. 2020;12:e8632-e. doi:10.7759/cureus.8632

19. Uribarri A, Núñez-Gil IJ, Aparisi A, Becerra-Muñoz VM, Feltes G, Trabattoni D, et al. Impact of renal function on admission in COVID-19 patients: an analysis of the international HOPE COVID-19 (Health Outcome Predictive Evaluation for COVID 19) Registry. J Nephrol. 2020;33:737-45. doi:10.1007/s40620-020-00790-5

20. Eltzschig HK, Carmeliet P. Hypoxia and inflammation. N Engl J Med. 2011;364:656-65. doi:10.1056/NEJMra0910283

21. Del Vecchio L, Locatelli F. Hypoxia response and acute lung and kidney injury: possible implications for therapy of COVID-19. Clin Kidney J. 2020;13:494-9. doi:10.1093/ckj/ sfaa 149

22. Shu S, Wang Y, Zheng M, Liu Z, Cai J, Tang C, et al. Hypoxia and hypoxia-inducible factors in kidney injury and repair. Cells. 2019;8:207. doi:10.3390/cells8030207

23. Tang N, Li D, Wang X, Sun Z. Abnormal coagulation parameters are associated with poor prognosis in patients with novel coronavirus pneumonia. J Thromb Haemost. 2020;18:844-7. doi:10.1111/jth.14768

24. Zhou F, Yu T, Du R, Fan G, Liu Y, Liu Z, et al. Clinical course and risk factors for mortality of adult inpatients with COVID-19 in Wuhan, China: a retrospective cohort study. Lancet. 2020;395:1054-62. doi:10.1016/S01406736(20)30566-3

25. Tan YK, Goh C, Leow AST, Tambyah PA, Ang A, Yap ES, et al. COVID-19 and ischemic stroke: a systematic review and meta-summary of the literature. J Thromb Thrombolysis. 2020;50:587-595. doi: 10.1007/s11239-020-02228-y

26. Rahman MH, Zahan MS, Al Hasib T, Ahmed KA, Khanam $\mathrm{M}$, Omit MS, et al. Current knowledge on mechanisms involved in SARS-CoV-2 infection and kidney diseases. J Adv Biotechnol Exp Ther. 2020;3:30-5.

27. Favalli EG, Ingegnoli F, De Lucia O, Cincinelli G, Cimaz R, Caporali R. COVID-19 infection and rheumatoid arthritis: Faraway, so close! Autoimmun Rev. 2020;19:102523. doi:10.1016/j.autrev.2020.102523

28. Mukherjee A, Ghosh R, Aftab G. Rhabdomyolysis in a patient with coronavirus disease 2019. Cureus. 2020; 12:e8956-e. doi:10.7759/cureus.8956

29. Petejova N, Martinek A. Acute kidney injury due to rhabdomyolysis and renal replacement therapy: a critical review. Crit Care. 2014;18:224. doi:10.1186/cc13897

30. Binois Y, Hachad H, Salem J-E, Charpentier J, LebrunVignes B, Pène F, et al. Acute kidney injury associated with lopinavir/ritonavir combined therapy in patients with Covid-19. Kidney Int Rep. 2020:10.1016/j.ekir.2020.07.035.

31. Adamsick ML, Gandhi RG, Bidell MR, Elshaboury RH, Bhattacharyya RP, Kim AY, et al. Remdesivir in patients with acute or chronic kidney disease and COVID-19. J Am Soc
Nephrol. 2020;31:1384-6. doi:10.1681/asn.2020050589

32. Grein J, Ohmagari N, Shin D, Diaz G, Asperges E, Castagna A, et al. Compassionate use of remdesivir for patients with severe COVID-19. N Engl J Med. 2020;382:2327-36. doi:10.1056/NEJMoa2007016

33. Puelles VG, Lütgehetmann M, Lindenmeyer MT, Sperhake JP, Wong MN, Allweiss L, et al. Multiorgan and renal Tropism of SARS-CoV-2. N Engl J Med. 2020;383:590-2. doi:10.1056/NEJMc2011400

34. Su H, Yang M, Wan C, Yi L-X, Tang F, Zhu H-Y, et al. Renal histopathological analysis of 26 postmortem findings of patients with COVID-19 in China. Kidney Int. 2020;98(1):219-27. doi:10.1016/j.kint.2020.04.003

35. Diao B, Wang C, Wang R, Feng Z, Tan Y, Wang H, et al. Human kidney is a target for novel severe acute respiratory syndrome coronavirus 2 (SARS-CoV-2) infection. medRxiv. 2020:2020.03.04.20031120. doi: 10.1101/2020.03.04.20031120

36. Werion A, Belkhir L, Perrot M, Schmit G, Aydin S, Chen $\mathrm{Z}$, et al. SARS-CoV-2 Causes a Specific Dysfunction of the Kidney Proximal Tubule. Kidney Int. 2020:S00852538(20)30912-1. doi:10.1016/j.kint.2020.07.019

37. Kormann R, Jacquot A, Alla A, Corbel A, Koszutski M, Voirin P, et al. Coronavirus disease 2019: acute Fanconi syndrome precedes acute kidney injury. Clin Kidney J. 2020;13:362-70. doi:10.1093/ckj/sfaa109

38. Cheng Y, Luo R, Wang K, Zhang M, Wang Z, Dong L, et al. Kidney disease is associated with in-hospital death of patients with COVID-19. Kidney Int. 2020;97:829-38. doi:10.1016/j.kint.2020.03.005

39. Chen N, Zhou M, Dong X, Qu J, Gong F, Han Y, et al. Epidemiological and clinical characteristics of 99 cases of 2019 novel coronavirus pneumonia in Wuhan, China: a descriptive study. Lancet. 2020;395:507-13. doi:10.1016/ S0140-6736(20)30211-7

40. Guan W-j, Ni Z-y, Hu Y, Liang W-h, Ou C-q, He J-x, et al. Clinical characteristics of coronavirus disease 2019 in China. N Engl J Med.2020;382:1708-20. doi:10.1056/ NEJMoa2002032

41. Yang X, Yu Y, Xu J, Shu H, Xia Ja, Liu H, et al. Clinical course and outcomes of critically ill patients with SARSCoV-2 pneumonia in Wuhan, China: a single-centered, retrospective, observational study. Lancet Respir Med. 2020;8:475-81. doi:10.1016/S2213-2600(20)30079-5

42. Hayat A, Majeed N, Abbas S, Akhtar F, Tashfeen S, Siddique A. Renal impairment: is there a need for monitoring renal functions in COVID infection. Pak Armed Forces Med J. 2020;70:S354-57.

43. Hirsch JS, Ng JH, Ross DW, Sharma P, Shah HH, Barnett $\mathrm{RL}$, et al. Acute kidney injury in patients hospitalized with COVID-19. Kidney Int. 2020;98:209-218. doi: 10.1016/j. kint.2020.05.006

Copyright (c) 2021 The Author(s); Published by Nickan Research Institute. This is an open-access article distributed under the terms of the Creative Commons Attribution License (http://creativecommons.org/licenses/by/4.0), which permits unrestricted use, distribution, and reproduction in any medium, provided the original work is properly cited. 